\title{
Non Linear and Multi Fractional Tuning Method for Autonomous Vehicles
}

\author{
Jerwinprabu $\mathrm{A}^{1^{*}}$ and Ashish Tuptee ${ }^{2}$ \\ ${ }^{1}$ Bharati Robotic Systems India Pvt Ltd Pune, India \\ ${ }^{2}$ Bharati Robotic Systems India Pvt Ltd Pune, India
}

Received: 12, November 2018; Accepted: 31, December 2018; Published: 04, January 2019

*Corresponding author: Jerwinprabu, Bharati Robotic Systems India Pvt Ltd Pune, India. E-mail: jerwinprabu@gmail.com

\begin{abstract}
The mathematical model of mover is determined using a NewtonEuler formulation. This paper deals with the simulation depend on proposed controller of an industrial mover that can overcome this trouble. It is important to notice that these control loop parameters error cannot be eliminated from the interaction system completely. Within these possibilities, the control system's performance and the robustness must be considered as important. The aim is to provide industrial oriented solutions and improve the behavior of a differential control system, with an Auto tuning Proportional-Integral-Derivative (ATPID) controller structure. Fractional Order Proportional Integral Derivative controller tuned by genetic algorithm, it is investigated to control and stabilize the position and attitude of control system using feedback linearization. This controller is used as a reference to compare its results with Proportional Integral Derivative (PID) controller tuned by gain margin. The control structure performance is evaluated through the response and minimizing the error of the position and attitude. Simulation results, demonstrates that position and attitude control using auto PID has fast response, better steady state error and measurement error than PID. By simulation the two controllers are tested under the same conditions using Simulink under Matlab programming.
\end{abstract}

Keywords: PID control; Auto Tuning Proportional Integral Derivative (ATPID); Tuning Methods; Optimality Degree; RegulatoryControl; Parametric Stability;

\section{Introduction}

Despite the advancement in the control principle area, PID-type controllers are still the majority regularly used control algorithm in industry. That is because of its design and implementation simplicity [11], there are four Shortcomings in classical PID control modelling process which are: oversimplification, error computation, and noise degradation in the derivative control and performance loss in the form of a linear weighted sum in the control law, also difficulties that can be done by the integral control. Most works have utilized Euler angles for modelling. Additionally it considers the dynamic models of rotors, gears and motors. But most of literature reviews didn't give the acceptable results compared to the required position and attitude where, the target of all controllers' techniques is to stabilize attitude and position of mover with better response. Considering that in mechanical process control applications, it is required a decent load-unsettling influence dismissal (generally known as administrative control), and also, a great transient reaction to target changes (It can be defined as angle control system), the process control configuration ought to think about the two potential outcomes of operation. Many techniques for this kind of PID controllers have been figured in the part of the most recent years [9] [16], Fractional calculus is an area of mathematics that deals with derivatives and integrals using noninteger orders. Fractional order derivatives and integrals have been used in industrial applications and different fields. In PID controller modelling process, the 5 parameters (kp, kd, $\mu$, ki and $\lambda$ ) require to be chosen depend upon some design specifications. In this way there is a require to an effective global methodology to optimize these parameters naturally. Gain Margin is one of evolutionary optimization strategies used to optimize the 5 parameters of the PID controller. Movers are motivating platform for Automated Guided Vehicle Robotics research. The thriving interest of autonomous robots in military, farming, mining, fire fighting and remote sensing and so on has given great impetus to controller research and improvement in this field. The research in controller design of mover is as yet having troubles because of high maneuverability, system nonlinearity, and strongly coupled multivariable and under-actuated condition. In a synthetic teaching environment, whereby students taught cyber students algebra concepts, participants were extrinsically motivated to compete with cyber-partners, as long as the partners were weaker than the tutors. Performance increased, however, learning goals of mathematics conceptual learning were not met. The controller output is straightforwardly fed into the dynamic model without making any mapping in the actuator space. In the simulations presented here, the thrust input cannot be more than double the weight of the matrix; similarly a suitable threshold is additionally put in the torque input. These thresholds have been put to make the control laws as practical as possible. 


\section{Related work}

There are a several literature reviews of mover control for upgraded performance such as classical linear methodologies for example automated tuning PID [10]. Likewise, extraordinary advances on ideal techniques in view of settling PID arrangements have been accomplished [18]. However, these strategies, albeit powerful, use to depend on to some degree complex numerical enhancement systems and don't give auto-tuning regulation. Rather, the tuning of the process control is characterized as the arrangement of the streamlining issue. In addition, now and again the strategies considered just the framework execution [4], or its heartiness [2] [6] [3]. In any case, the most intriguing cases are the ones that consolidate execution and power, since they look with every one of framework's necessities [12]. The past referred to strategies think about the execution and strength together in the control plan [14] [17]. Be that as it may, nobody treats particularly the execution/strength exchange off issue, nor consider in the definition the servo/direction exchange off or the communicating between these factors.

\section{Implementation of proposed model}

The time-independant version (in three independent (space) variables is called the Laplacian operator. When it's action on a function or vector vanishes, the resulting equation is called the wave equation (or Laplace's equation). When it's action is identified with a non-zero function (or vector function) the resulting equation is called Poisson's equation. This equation is fundamental to and of great importance in field theory. The factors of intrigue could be portrayed as takes after:

This model is usually utilized as a part of process control on the grounds that is basic and portrays the elements of numerous modern procedures roughly [11]. Process control system is done to adapt the total angular momentum of the rotation; otherwise the machine will lost the control, it will try to move out of control. The Mover has differential and steering wheel control system. Hence, Movers are going to move in a particular direction or differential direction, it will calculate the movement path. A low level controller balances out the rotational speed of each pulsus, to keep up the requirement for site experimentation is an actual moment that thinking about the modern use of a system appeared in [Figure.1]. The Mover can perform angular movement with Steering Geometry Interaction System [15], it make slow precise movements. The differential wheel drive system give a higher payload capacity. Movers are moderately less difficult because they don't bring convoluted swash plates and linkages. There are some states that we require in ground vehicle recorded as: Estimation State, calculate position, load disturbance, control tuning and velocity of mover. Control, drive motors and delivers desired actions in order to navigate to the desired state. Mapping, the mover must have basic ability to map its environment. Planning: Finally, the mover must be able to track the trajectory planning and load unsettling influence reactions gives the PID parameters, tuning range appeared in [Table I] and[ Figure.2.] $\tau$ indicates the tuning range for the various positions. $\mathrm{EP}$ represent the end point, where the tuning parameter will reach for destination. LD indicates the linear direction of the bot, it will help to position identification and tuning ratio.

It demonstrates the execution of the two different function when the system control process is working in linear direction, system and direction mode. In this manner, the tuned Performance integral action is varied appeared in [Table 2]. From a worldwide perspective, it will appear to be smarter to pick the tuning-point settings.

$\mathrm{E}(\mathrm{S})$

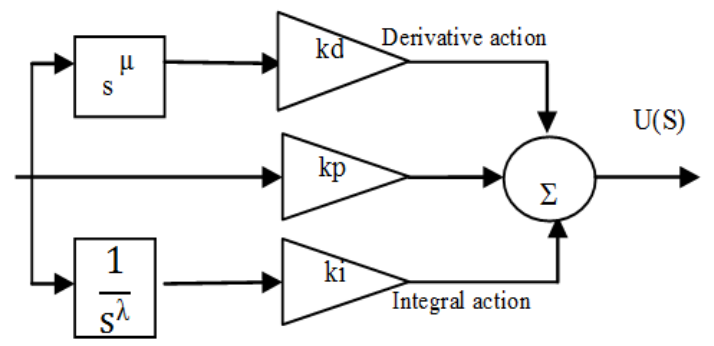

Figure 1: Feedback Systematic Process control system.

Table 1: PID Tuning Range Settings for (Sp) and (LD)

\begin{tabular}{|c|c|c|c|c|}
\hline$\tau$ range & \multicolumn{2}{|c|}{ 0.5 - 1.5 } & \multicolumn{2}{c|}{$\mathbf{1 . 5}-\mathbf{2}$} \\
\hline Tuning & EP & LD & EP & LD \\
\hline $\boldsymbol{a 1}$ & 1.436 & 1.492 & 1.97 & 1.924 \\
\hline $\boldsymbol{b 1}$ & -0.670 & -0.966 & -0.76 & -0.735 \\
\hline $\boldsymbol{a} \mathbf{2}$ & 1.342 & 1.125 & 1.54 & 1.767 \\
\hline $\boldsymbol{b} \mathbf{2}$ & -0.765 & -0.753 & -0.33 & -0.921 \\
\hline $\boldsymbol{a 3}$ & 0.892 & 0.975 & 1.68 & 1.561 \\
\hline $\boldsymbol{b 3}$ & 0.995 & 0.998 & 0.56 & 0.763 \\
\hline $\boldsymbol{a 1}$ & 1.436 & 1.492 & 1.97 & 1.924 \\
\hline $\boldsymbol{b 1}$ & -0.670 & -0.966 & -0.76 & -0.735 \\
\hline
\end{tabular}

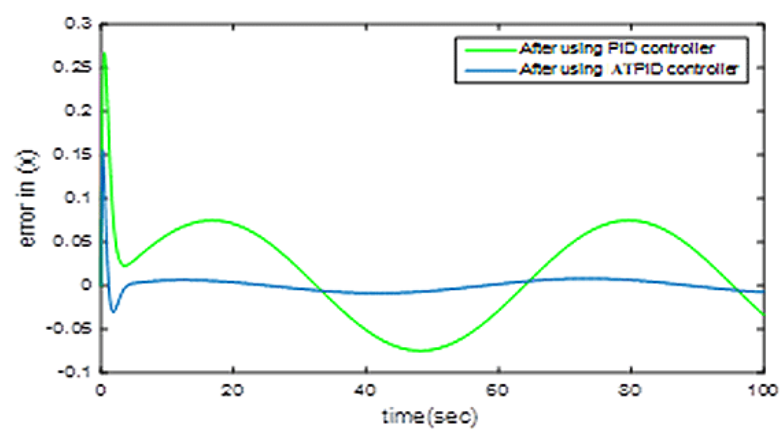

Figure 2: Execution example - Responses for servo access control and regulation for control system Auto tuning PID. 


\begin{tabular}{|c|c|c|c|}
\hline \multicolumn{2}{|c|}{ Table2: P1 load-disturbance tuning } \\
\hline Auto-tuning & $\boldsymbol{K}_{\boldsymbol{p}}$ & $\boldsymbol{K}_{\boldsymbol{i}}$ & $\boldsymbol{K}_{\boldsymbol{d}}$ \\
\hline end point(ep) & 1.767 & 1.351 & 0.513 \\
\hline load disturbance(Id) & 2.638 & 1.012 & 0.559 \\
\hline Control tuning & 1.563 & 0.994 & 1.735 \\
\hline
\end{tabular}

The forces following up on the framework are the thrusts J from each of the rotor and the force of gravity Jsp. The moments following up on the framework are the moments due to each of the thrust and the drag moment Jld which is created because of the propeller rotation.

$$
\begin{array}{llll}
\operatorname{Jsp}(\mathrm{sp}) & \leq & \operatorname{Jsp}(\mathrm{ld}) & (1) \\
\operatorname{Jld}(\mathrm{ld}) & \leq & \operatorname{Jld}(\mathrm{sp}) & (2)
\end{array}
$$

Subsequently, we have determined $\mathrm{x} \in\{s p, l d\}$ and $\mathrm{z} \in\{s p, l d\}$, sp represents for end-point (servo control system) auto-tuning and $l d$ for stack unsettling influence (controller) tuning.

Execution won't be ideal for the two circumstances. The dynamics of a mover by using the Newton-Euler formalism. The inspiration is gotten from Mellinger work. where, $\tau$ is the net torque, $F$ is the net force acting on the mover, a is the linear acceleration of the center of mass, $\mathrm{I}_{3}$ is a $3 \times 3$ identity matrix called the moment of inertia, $\omega$ is mover velocity angle, $v$ is the linear velocity, $m$ is the mass and $\alpha$ is the acceleration angle. On this premise we characterize a process control parameter $\gamma$

$$
\gamma=\left[\gamma_{1}, \gamma_{2}, \gamma_{3}\right]
$$

The qualities of this factor are limited to $\gamma \in[0,1.5] \mathrm{I}=1$, $2.5,3$. Additionally, the end-point autotuning will relate a shape imperative for each $\gamma \mathrm{i}=0$, while the heap unsettling influence tuning compares to $\gamma \mathrm{i}=1.5$. The controller settings family $\left[K_{p}(\gamma 1)\right.$, $\left.\mathrm{T}_{\mathrm{i}}(\gamma 2), \mathrm{T}_{\mathrm{d}}(\gamma 3)\right]$, can be communicated, in a more broad shape, as

$$
\begin{array}{ll}
\operatorname{Kp}(\gamma 1) & =\mathrm{fKp}(\gamma 1 ; \text { Kpld,Kpsp }) \\
\operatorname{Ti}(\gamma 2) & =\mathrm{fTi}(\gamma 2 ; \text { Tild,Tisp }) \\
\operatorname{Td}(\gamma 3) & =\mathrm{fTd}(\gamma 3 ; \text { Tdld,Tdsp })
\end{array}
$$

where $\gamma \mathrm{i} \in[0,1.5] \mathrm{I}=1,2.5,3$ and $\left[K_{p s p} T_{i s p^{\prime}} T_{d s p}\right]$ and $\left[K_{p l p^{\prime}} T_{i l p^{\prime}} T_{d l d}\right]$ remain for the end-point and process system-unsettling influence settings for $\left[K_{p} T_{p} T_{d}\right]$ individually. Additionally, every $\gamma \mathrm{i}$ change needs to fulfill the shape imperatives with the frame

$\begin{array}{ll}\text { Tild }= & \text { fTi(1;Tild,Tisp) } \\ \text { Tdsp }= & \text { fTd(0;Tdld,Tdsp) } \\ \text { Tdld }= & \text { fTd(1;Tdld,Tdsp) }\end{array}$

Taking (5) as the shape imperatives detailing, Where, $I=$ Moment of inertia, $r=\left[\mathrm{x} \mathrm{y} \mathrm{z}^{\mathrm{T}}\right.$, [p q r] the body angular Velocities, $m=$ the system Mass. The relationship between the rate of change of $(\varphi, \theta$ and $\psi)$ and body angular velocities is,

$$
\begin{array}{ll}
\mathrm{T}_{\mathrm{i}}(\gamma 2) & =\gamma 2 \mathrm{~T}_{\text {ild }}+(1-\gamma 2) \mathrm{T}_{\text {isp }} \\
\mathrm{T}_{\mathrm{d}}(\gamma 3) & =\gamma 3 \mathrm{~T}_{\mathrm{dld}}+(1-\gamma 3) \mathrm{T}_{\mathrm{dsp}}
\end{array}
$$

Here, the goal is to present the steadiness examination of the geometry angle produced by the controller characterized by (7) regarding the vector $\gamma$. In the first place, consider that the PID controller is communicated with its three picks up as,

$$
K_{c}=K_{p}, K_{i}=K_{p} / T_{i} K_{d}=K_{p} T_{d}
$$

The transitional controller given by (7) asymptotically balances out the framework gave that the outskirt esteems are given and Table II, $1 / \mathrm{N}$ is adequately little and $\gamma \mathrm{i} \in[0,1.5]$ for I $=1,2.5$, 3. It means presented above, we demonstrate that the proposed fringe esteems for $\mathrm{K}_{c^{\prime}}$ in particular, $\mathrm{K}_{\mathrm{p}^{\prime}}, \mathrm{K}_{\mathrm{i}^{\prime}}, \mathrm{K}_{\mathrm{d}}$ ensuring then the presence of a settling PID controller. For each estimation of $\tau$ $\in[0.5,1.5] \cup[1.5,2]$, [Figure. 3] demonstrates the most extreme permitted corresponding addition and alternate increases given by the tuning conditions for the fringe parameters.

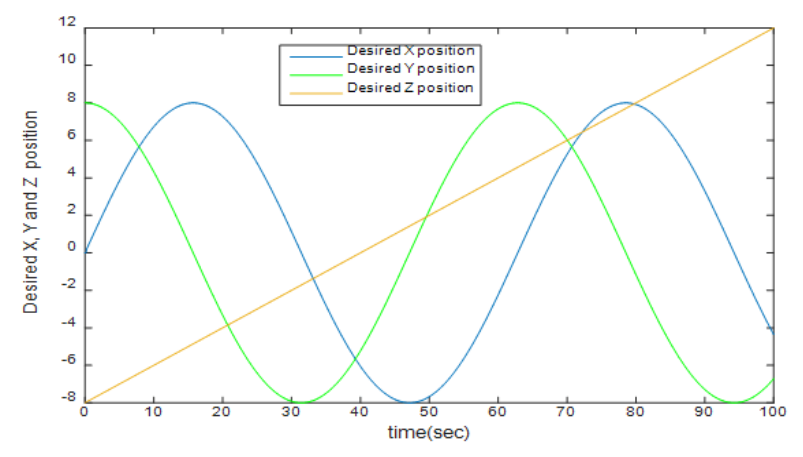

Figure 3: Proportional gain and slope values obtained by desired position parameters

As appeared in the block PID controller is inner loop while position controller is the outer loop. It is sensible to perceive that the dynamics of the inner loop must be quicker than the dynamics of the outer loop. [7]. [Figure. 4] demonstrates the stabilizing portion of the system and the polygon for the axial variation demonstrated in equation 9.

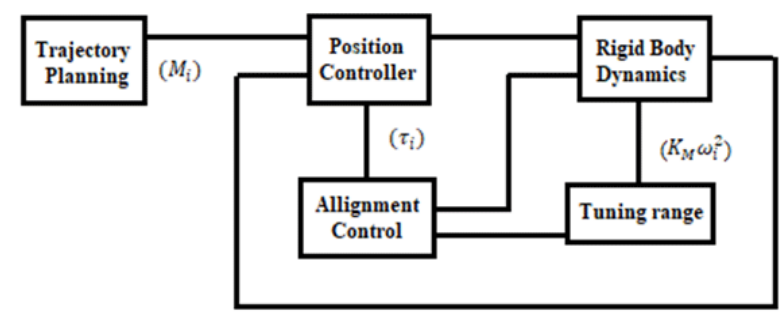

Figure 4: The trajectory planner of the system and the rigid body dynamics axial $\gamma$ variation.

$$
M_{i}=\tau_{i}=K_{M} \omega_{i}^{2}
$$

Motor Torques $\left(M_{i}\right)$ and Drag Torque $\left(\tau_{i}\right)$ (They have same magnitude however inverse signs $\left.\left(\mathrm{K}_{\mathrm{M}} \omega_{\mathrm{i}}^{2}\right)\right)$ :

It is sensible to perceive that the dynamics of the inner loop must be quicker than the dynamics of the outer loop. In hover 
arrangements the dynamics of attitude do not matter much in general, however in situations where the robot needs to make maneuvers, it is essential to have a quicker reposition controller [2]. The point of PID is to design a position and stabilisation controller of a mover by choice of a PID parameters gains $\left(\mathrm{k}_{\mathrm{p}}, \mathrm{k}_{\mathrm{d}}\right.$ and $\mathrm{k}_{\mathrm{i}}$ ) utilizing gain margin, where gain margin is an optimization method rely upon the mechanisms of regular selection in the angular movement [8]. Be that as it may, $\alpha$ parameter permits to settle on a more broad decision for the inclination of the framework operation (not just considering the ideal opportunity for every operation mode) [3]. This control law works well under hover conditions. According to equation 10 and 11 linearizing the dynamic model at the hover configuration, where the system model is reduced in different force.

$$
m \ddot{r}=\left[\begin{array}{c}
0 \\
0 \\
-m g
\end{array}\right]+R_{B}^{w}\left[\begin{array}{c}
0 \\
0 \\
F_{1}+F_{2}+F_{3}+F_{4}
\end{array}\right]
$$

The equation of angular Motion is:

$$
I\left[\begin{array}{c}
\dot{p} \\
\dot{q} \\
\dot{r}
\end{array}\right]=\left[\begin{array}{c}
L\left(F_{2}-F_{4}\right) \\
L\left(F_{3}-F_{1}\right) \\
M_{2}+M_{4}-M_{1}-M_{3}
\end{array}\right]-\left[\begin{array}{c}
p \\
q \\
r
\end{array}\right] * I\left[\begin{array}{c}
p \\
q \\
r
\end{array}\right]
$$

Where, $\mathrm{I}=$ Moment of inertia, $\mathrm{r}=[\mathrm{xyz}]^{\mathrm{T}},[p q r]$ the body angular Velocities, $\mathrm{m}=$ the system Mass. The relationship between the rate of change of $(\varphi, \theta$ and $\psi)$ and body angular velocities is:

$$
\left[\begin{array}{c}
p \\
q \\
r
\end{array}\right]=\left[\begin{array}{ccc}
c_{\theta} & 0 & -c_{\varphi} s_{\theta} \\
0 & 1 & s \varphi \\
s_{\theta} & 0 & c \varphi c \theta
\end{array}\right]\left[\begin{array}{c}
\dot{\varphi} \\
\dot{\theta} \\
\cdot
\end{array}\right]
$$

\section{Optimization Problem Formulation}

Gain margin applied for tuning PID gains $\mathrm{kp}$, kd and ki for the three position $(\mathrm{x}, \mathrm{y}$ and $\mathrm{z}$ ) utilizing Integral Square-Error to guarantee ideal control performance at nominal operating conditions. The Three gains of PID after tuning for $\mathrm{X}\left(\mathrm{k}_{\mathrm{p} 1}=5.45 \mathrm{and}\right.$ $\mathrm{k}_{\mathrm{d} 1}=2$, $\left.\mathrm{ki}=3.1\right)$, for $\mathrm{Y}\left(\mathrm{k}_{\mathrm{p} 2}=1.562, \mathrm{k}_{\mathrm{d} 2}=6.5994, \mathrm{k}_{\mathrm{i}}=4.5864\right)$ and for $\mathrm{Z}$ $\left(\mathrm{k}_{\mathrm{p} 3}=13.96278, \mathrm{k}_{\mathrm{d} 3}=5.2511, \mathrm{k}_{\mathrm{i}}=48.45696\right)$ at that point alter this error signal to give control input for system. The control input then forces the system to deliver output as close as possible to the desire position.

[13] [5] Fractional Order Calculus is a generalization of the conventional integration and differentiation that include noninteger orders. This will calculate the target point movement ratio. Fundamental operator representing the fractional-order differential and integration is presented in [Figure. 5]. Linear operator Jd was translated as integrator when ' $a$ ' is negative and differentiator when 'a' is positive. Something else, $J_{d}$ is a unity when a is zero. The most widely recognized form of a ATPID controller is the $\mathrm{PI}^{\lambda} \mathrm{D}^{\mu}$ controller. Including an integrator of order $\lambda$ and a differentiator of order $\mu$ where $\lambda$ and $\mu$ can be any real numbers.

$$
G_{c}(s)=\frac{U(s)}{E(s)}=k_{p}+k_{I} \frac{1}{s^{\lambda}}+k_{D} s^{\mu},(\lambda, \mu>0)
$$

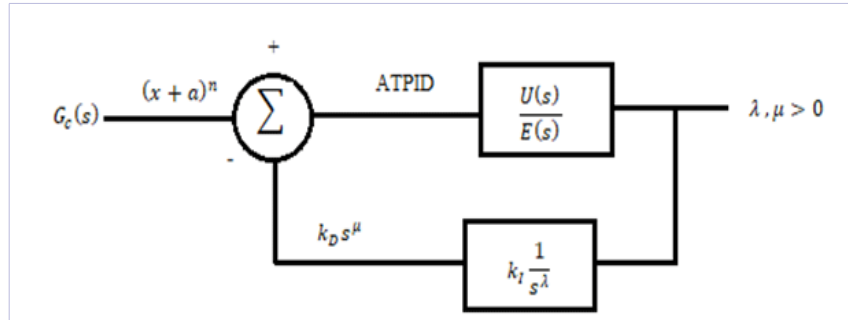

Figure 5: The stabilizing portion of the system and the polygon for the axial $\gamma$ variation.

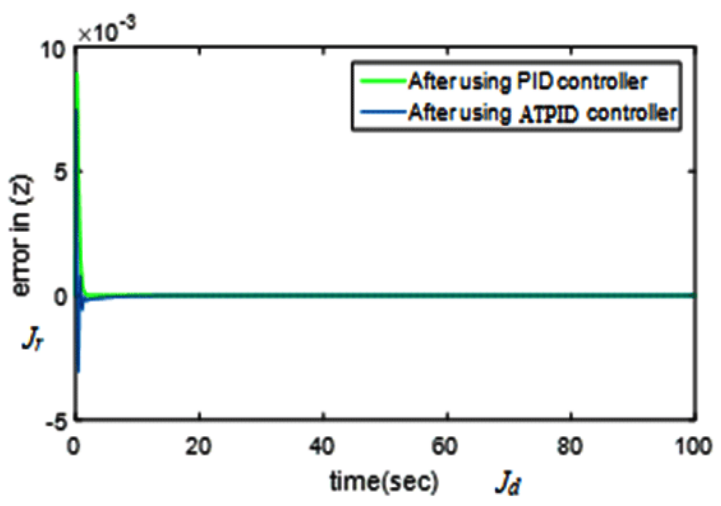

Figure 6: Plane $\mathrm{J}_{\mathrm{r}}-\mathrm{J}_{\mathrm{d}}$

Where $G c(s)$ is the transfer function of the controller, $E(s)$ is the error, and $U(s)$ is controller's output. The control signal $u(t)$ can then be expressed in the time domain as:

$$
u(t)=k_{p} e(t)+k_{I} D_{t}^{-\lambda} e(t)+k_{D} D_{t}^{\mu} e(t)
$$

Where, choosing $\lambda=1$ and $\mu=1$, a traditional PID controller can be recovered. The choosing of $\lambda=1, \mu=0$, and $\lambda=0, \mu=1$ respectively corresponds traditional PI \& PD controllers.

In Fig. 6, the record is spoken to by the bolt between the "perfect" point and the comparing to the middle of the road autotuning (plane $\mathrm{J}_{\mathrm{r}}-\mathrm{J}_{\mathrm{d}}$ ). It can be expected that the $\mathrm{PI}^{\lambda} \mathrm{D}^{\mu}$ controller may upgrade the systems efficiency. Control of industrial systems is one of the most important features of the $\mathrm{PI}^{\lambda} \mathrm{D}^{\mu}$ controller. Another feature lies in the fact that $\mathrm{PI}^{\lambda} \mathrm{D}^{\mu}$ controllers are very low sensitive and accurate. If the starting point having a fixed value, followed by that value end point coordinate calculation will get configured automatically. Fig. 7 demonstrates accomplishment of claimed robustness level. ATPID optimized by gain using Integral Square Error cost function to ensure ideal control efficiency at nominal operating conditions. Where, each ATPID controller has 5 parameters, there are totally 15 parameters to be optimized by gain. PID tuned by position estimation and gain where, the controllers tried to track the path of a helical trajectory. Starting from random initialized parameters, gain margin progressively minimizes various integral performance indices iteratively while finding optimal set of parameters for the ATPID and PID controller. The algorithm calculation ends if the estimation value of the objective function does not change obviously over some progressive iterations. 
The values of the 9 PID parameters obtain by y axis with fitness value 0.025411 after 340 epochs. On the off chance that we utilize the data of [Figure. 7] and [Figure. 8], and the preprocessing case as the beginning stage, that is conceivable to notice that for each level.

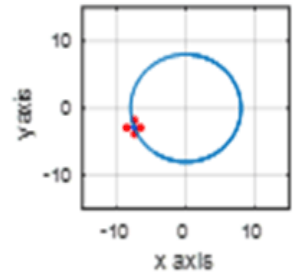

Figure 7: The accomplishment of claimed robustness level

ATPID control provides the mover with minimum error between desired and actual position for (X, Y and Z) respectively compared with PID controller as presented in [Figures 8, 9 and 10]. Where, gain reaches to the values of the 15 ATPID parameters after 46 epochs with fitness 0.404491 .

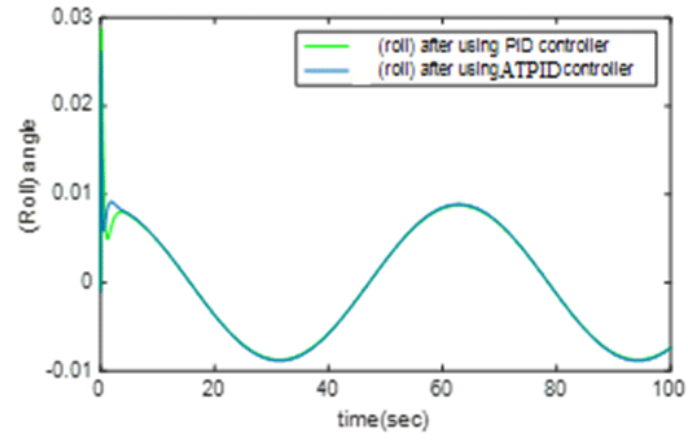

Figure 8: Combined roll axis for each robustness level tuning

\section{Result and Discussion}

In process control output displayed in [Figure.9], it is critical to promise some level of heartiness, By comparing steady state and RMS error in a system it was found that the ATPID's errors (Steady State error for X position $=-0.003438, \mathrm{Y}=0.0013449, \mathrm{Z}=-$ $2.66^{*} 10^{-15}$ and RMS error $=0.00012$ ) less than PID's errors (Steady State error for $\mathrm{X}=-0.03367, \mathrm{Y}=-0.06726, \mathrm{Z}=6.217^{*} 10^{-15}$ and RMS error $=0.00695$ ). ATPID controller has fast response and small errors for the required position of quad rotor. [Figures7, 8 and 9] give complete comparisons between the various controllers for $\mathrm{X}, \mathrm{Y}$ and $\mathrm{Z}$ errors respectively. The error calculation depends upon the stored end point value and actual value. We search for a general condition as,

$$
u(t)=k_{p} e(t)+k_{I} D_{t}^{-\lambda} e(t)+k_{D} D_{t}^{\mu} e(t)
$$

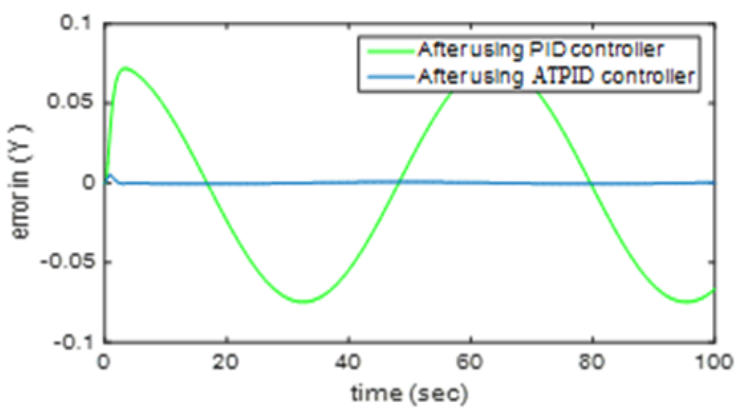

Figure 9: Process Control - Linear method $(\sigma=0.50)$

Also alignment position angles $(\operatorname{roll}(\Phi) \operatorname{pitch}(\theta)$ yaw $(\psi))$ after using ATPID has fast response and small errors for the required orientation than controlled based on PID tuned by calculated error value and gain as shown in [Figures10, 11 and 12].

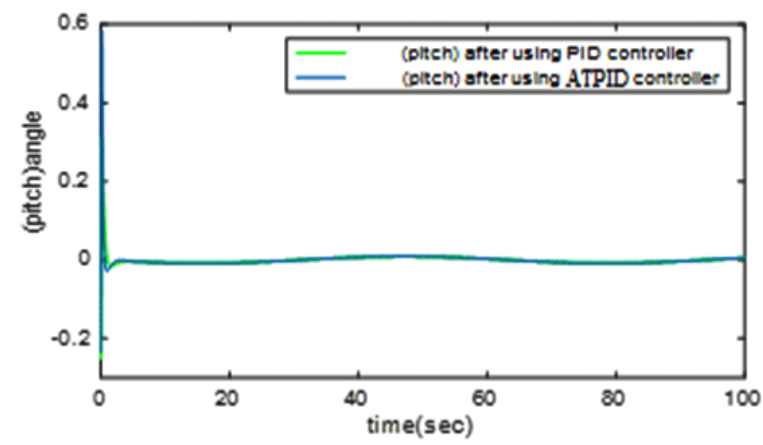

Figure 10: Process control - Generic response and debasement case

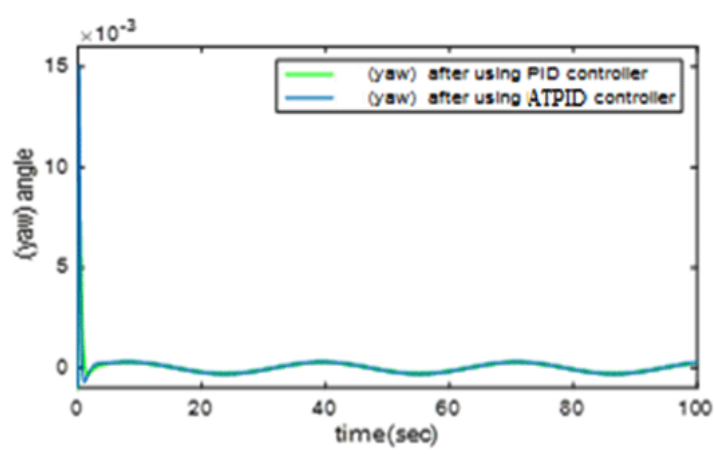

Figure 11: The accomplishment of the fixed degradation level tuning. 


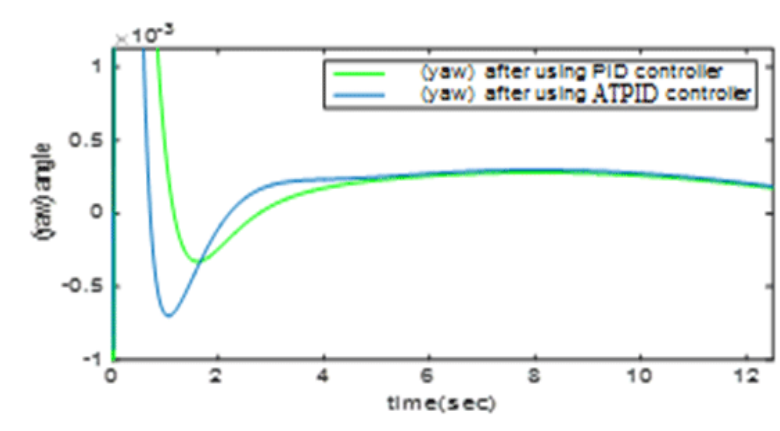

Figure 12: Variation of the index yaw value set

\section{Conclusion}

In this work, ATPID controllers have been used to position and movement control of mover to achieve the required position with fast response and minimum error. As appeared in results ATPID technique compared with PID tuned using gain, so from the simulation results it was concluded that: By comparing steady state and RMS error the position control of the $\mathrm{X}, \mathrm{Y}$ and $\mathrm{Z}$ controlled using ATPID has better performance, steady state error and RMS error than controlled using PID. The position estimation angle responses had showed to us that the system designed based on ATPID controller has much faster response than using the PID controller.

\section{References}

1. M Odom. Segregated Classes for the Retarded. Educational Considerations. 1976;3(3).

2. Tuning of PID controllers based on gain and phase margin specifications. Control Engineering Practice. 1994;2(6):1066.

3. H.-W Fung, Q G Wang, T H Lee. PI Tuning in Terms of Gain and Phase Margins. Automatica. 1998;34(9):1145-1149.

4. D Chen, DE Seborg. PI/PID Controller Design Based on Direct Synthesis and Disturbance Rejection. Industrial \& Engineering Chemistry Research. 2002;41(19):4807-4822.

5. M Chidambaram, R P Sree. A simple method of tuning PID controllers for integrator/dead-time processes. Computers \& Chemical Engineering. 2003;27(2):211-215.
6. L Bascetta, A Leva. FIR based causal design of 2-d.o.f. controllers for optimal setpoint tracking. Journal of Process Control. 2008;18(5):465478.

7. Lars-ola Bligard, Asa Fast-Berglund, Sandra Mattsson. Finding Trends in Human-Automation Interaction Research in Order to Formulate a Cognitive Automation Strategy for Final Assembly. International Journal of Advanced Robotics and Automation. 2016;1(1).

8. Arrieta, A Ibeas, R Vilanova. Stability analysis for the intermediate servo/regulation PID tuning. IEEE Conference on Emerging Technologies \& Factory Automation. 2009.

9. Process Identification Methods for Discrete-Time Difference Equation Models. Process Identification and PID Control. 2009:317-336.

10.E Joelianto. Robust Ho PID Controller Design Via LMI Solution of Dissipative Integral Back stepping with State Feedback Synthesis. Robust Control, Theory and Applications. 2011.

11. M Shamsuzzoha, M Lee. IMC Filter Design for PID Controller Tuning of Time Delayed Processes. PID Controller Design Approaches - Theory, Tuning, and Application to Frontier Areas. 2012.

12. A J Prabu, J Narmadha, K Jeyaprakash. Artificial Intelligence Robotically Assisted Brain Surgery. Artificial Intelligence. 2014;4(5).

13. Prabu A Jerwin. Design Optimisation of Cam Shaft Angle Monitoring System For Industrial Improvements. IOSR 2015;5:29-36.

14. G Deshmukh, C Kadu. Design of two degrees of freedom PID controller for temperature control system. International Conference on Automatic Control and Dynamic Optimization Techniques (ICACDOT). 2016.

15. Jerwin Prabu A, Abhinav Tiwari. Optimization approach for a Climbing robot with target tracking in WSNs. Journal of Ocean Engineering and Science. 2018;3(4):282-287.

16. Sudha, S N Deepa. Optimization for PID Control Parameters on Pitch Control of Aircraft Dynamics Based on Tuning Methods. Applied Mathematics \& Information Sciences. 2016;10(1):343-350.

17.V C A D M Alfaro, R Vilanova. Two-Degree-of-Freedom PID Controllers Structures. Model-Reference Robust Tuning of PID Controllers Advances in Industrial Control. 2016:7-19.

18. Kaleeswari N, Prabu, S S A J. Optimization Approach for Energy Consumption in Wireless Sensor Networks using Delay Aware Dynamic Routing Protocol. 2017. 